\title{
BIMFlexi-VR: A Virtual Reality Framework for Early-Stage Collaboration in Flexible Industrial Building Design
}

\author{
Iana Podkosova ${ }^{1 *}$, Julia Reisinger ${ }^{2}$, Hannes Kaufmann ${ }^{1}$ and Iva Kovacic ${ }^{2}$ \\ ${ }^{1}$ Faculty of Computer Science, Institute of Visual Computing and Human-Centered Technology, TU Wien, Vienna, Austria, \\ ${ }^{2}$ Faculty of Civil Engineering, Institute of Interdisciplinary Construction Process Management, TU Wien, Vienna, Austria
}

\section{OPEN ACCESS}

Edited by: Krzysztof Walczak,

Poznań University of Economics and Business, Poland

Reviewed by: Julia Ratajczak-Jeziorska, Budimex, Poland

Dionisis Andronas,

University of Patras, Greece Jakub Flotyński,

Poznań University of Economics and

Business, Poland

*Correspondence:

lana Podkosova

yana.podkosova@tuwien.ac.at

Specialty section:

This article was submitted to Virtual Reality in Industry,

a section of the journal

Frontiers in Virtual Reality

Received: 23 September 2021 Accepted: 04 February 2022 Published: 25 February 2022

Citation: Podkosova I, Reisinger J, Kaufmann H and Kovacic I (2022) BIMFlexi-VR: A Virtual Reality Framework for EarlyStage Collaboration in Flexible Industrial Building Design. Front. Virtual Real. 3:782169. doi: 10.3389/frvir.2022.782169
Integrated industrial building design is an interdisciplinary task, in which planning of flexible building structures requires effective communication and collaboration between all stakeholders already in early design stage. This paper presents BIMFlexi-VR, a collaborative framework which implements a real-time bidirectional link between a parametric modelling component created in Grasshopper for Rhinoceros that performs optimized structural calculations of an industrial building, and an immersive Virtual Reality environment in which the automatically calculated building is visualized. Users of BIMFlexiVR are able to change parameters defining the outcome of the structural calculation directly inside the virtual environment and see the modified building design together with the associated fitness metrics in a matter of seconds. Providing an efficient and intuitive platform for early exploration of industrial building designs, BIMFlexi-VR enables collaborative decision making and facilitates the creation of more efficient and sustainable industrial constructions.

Keywords: virtual reality, collaborative BIM, parametric modeling in VR, VR in AEC, VR in Industry 4.0, collaborative VR

\section{INTRODUCTION}

In Integrated Industrial Building Design, the successful implementation of each project is based on collaborative decision making and coupling of tools of multiple domain stakeholders-architects, engineers, production system planners and building owners. Traditionally, design of an industrial building is a lengthy process where workflows of individual stakeholders are disconnected, with their goals often conflicting and communication flows ineffective. In particular, structural parameters are often considered too late in the planning process (Reisinger et al., 2021b). As a result, industrial buildings suffer from suboptimal floorplans and load-bearing structures, making it impossible to adapt to future changes in production-once a building has been designed for a certain production type it cannot be used for other production types, as the structural composition of the building does not allow changes (Zhao and Tseng, 2003). Frequently, new construction becomes necessary increasing the overall economic expenditure and environmental impact of such projects.

In the research project BIMFlexi, a framework for integrated design of flexible industrial buildings is developed. In the context of the project, flexibility is defined as "the ability of the building structure to resist and adapt to changes in use through changing manufacturing conditions" (Reisinger et al., 2021a). Following this definition, the BIMFlexi design approach aims to create industrial building 
design solutions that can accommodate a variety of prioritized production plans. To achieve this goal, two major underlying problems in integrated industrial building design (Reisinger et al., 2021a) are addressed-1) lack of interoperability between discipline-specific tools and 2) poor communication workflows in the early design stage.

A wealth of new digital tools offer an excellent potential to support integrated industrial building design. While technologies such as Building Information Modeling (BIM), algorithmic modeling and collaborative Virtual Reality (VR) cannot be called emerging any longer, their power is not fully exploited by the practitioners of industrial building design yet. BIMFlexi offers a framework that uses these new digital technologies to address the problems stated above. BIM, parametric structural design and multi-objective optimization are combined to integrate production planning into building design, making design outcomes efficiently adaptable to rapidly changing production processes.

The focus and main contribution of this paper is BIMFLexiVR, a Virtual Reality-based communication and decision-making platform that enables early-stage communication between multidisciplinary stakeholders in the BIMFlexi project. The platform is implemented in Unity3D game engine that is coupled in real-time with an automated background optimization process that considers parametric structural design and production layout planning at the same time, providing a flexible building solution that can accommodate a selection of several prioritized production plans. This structural calculation and optimization environment is created with Grasshopper for Rhinoceros. BIMFlexi-VR establishes a bidirectional link between the visualization system and the structural calculation environment, allowing stakeholders to change parameters of the structural calculation from within the immersive virtual scene in a collaborative way and observe changes in the outcomes of building design in real-time already in the early stages of the planning process. This way, the limitations posed by different planning components and potential compromises can be easily explored by all involved parties. While the detailed description of the parametric structural design and optimization process is beyond the scope of this paper [it is presented in a previous publication, Reisinger et al. (2020)], the real-time connection between the structural calculation and optimization and the collaborative VR visualization component is crucial to the global workflow of BIMFlexi. It is presented in detail here. Alongside the description of the architecture and functionality of BIMFlexiVR, its first evaluation by teams of collaborating architects and structural engineers is presented which provides important insights not only for further improvement of the system but for the practices of using BIM and VR in integrated industrial building design in general.

\section{RELATED WORK}

The potential of the VR technology for the architecture, engineering and construction (AEC) has been understood already in the early days of immersive technologies research (Warwick et al., 1993; Whyte, 2003; Bouchlaghem et al., 2005; Fernandes et al., 2006). Indeed, VR technology allows to create immersive visualizations of constructions or room arrangements that do not exist yet, providing full-scale replicas that can be inspected by users from within. However, VR is not simply an advanced visualization platform. It allows users to become a part of the virtual world and to act upon it, as it allows them to share a common virtual environment in multi-user applications. For example, simulations in collaborative $\mathrm{VR}$ prove to be an effective tool for workspace analysis (Michalos et al., 2018). By exploiting the concepts of embodiment (Kilteni et al., 2012)providing a user with a virtual body that will serve as their representation in a shared environment and as the means of executing their effective agency in the virtual world, and strongly associated social presence (Slater et al., 1994; Lombard and Ditton, 1997; Oh et al., 2018) — the illusion of being together with others in that world, even if they are geographically distant, it is possible to create a simulation in which users behave and interact as if they were in a shared physical location. The possibility to explore virtual buildings by walking within them in a natural way is especially beneficial, allowing users to fully appreciate the scale of the construction and improving the sense of presence within the virtual building (Ruddle and Lessels,2009). With the latest advances in the VR technology, multiple affordable head-mounted displays (HMDs) include built-in tracking capabilities, making exploration by walking easily accessible to end users. The newest models such as Oculus Quest provide wireless solutions as well, increasing the ease of setup even further.

Whereas the applications of VR in architecture are numerous, we limit our overview to those that focus on BIM, and especially in conjunction with parametric structural design.

\subsection{BIM and Parametric Structural Design in VR}

Although solutions combining BIM and VR are still far from standard in the AEC industry, researchers and practitioners agree that the combined power of these technologies is beneficial, enhancing the ability of architects and structural engineers to communicate design decisions to team members (Sampaio,2018). Encouragingly, Anderson and colleagues found that even a desktop-based shared virtual world where members of a geographically distributed team were represented by avatars controlled via keyboard input proved to be better suited for within-team communication than BIM software where the $2 \mathrm{D}$ view of the floorplan was shared, although the latter was specifically designed for collaboration as well (Anderson et al., 2014). The authors propose that the advantage of the $3 \mathrm{D}$ environment over screen sharing consisted in the possibility to position avatars close to $3 \mathrm{D}$ elements being discussed as well as the ability of users to explore the model individually by moving their virtual viewpoints. Continuing their research on avatarmediated exploration of BIM by distributed AEC teams, authors established that avatar movement and positioning within a shared virtual environment provide helpful non-verbal cues, and further 
assisting the communication process when avatar actions map to usual social norms (Anderson et al., 2017). While the research was conducted in a desktop-based virtual environment, the findings make modern VR technology especially beneficial, since room-scale positional tracking of HMDs, and hand-held controllers result in the great freedom of movements for avatars, effortlessly allowing their precise positioning.

While the advantages of combining BIM and VR are evident, the necessity to transport $3 \mathrm{D}$ and meta information from BIM software into VR-supporting rendering engines has been the major difficulty in adopting BIM + VR solutions, often requiring an intermediate step of conversion to a $3 \mathrm{D}$ modelling format (Boton,2018; Wolfartsberger et al., 2018). Difficulties related to data conversion along with the incapability of many BIM + VR systems to show real-time changes of BIM models in VR were found to be among the principle limitations of examined solutions in the overview work of Sidani and colleagues (Sidani et al., 2021). Here, we provide an overview of previous research where this challenge has been met, paving the way to real-time data exchange between BIM and VR.

Early research on BIM + VR systems is focused on exploration of already finished, static models. For example, Johansson and colleagues presented a system in which a VR rendering engine is implemented as a plugin in the BIM-authoring software Revit (Johansson et al., 2014). The advantage of this system is the fact that designers do not need to convert their models from Revit format into other 3D modelling formats compatible with VR game engines. However, the plugin only allows to explore static models, although a changed design can be available for VR inspection in $20 \mathrm{~s}$. The user needs to remove the HMD every time they want to adjust the model in Revit. With the system using Oculus Rift DK, an early example of an affordable HMD without in-built position tracking, natural movements of users are limited to looking around while movement of the virtual camera is accomplished by pressing buttons on a connected remote control.

Focusing on improving communication between different stakeholders, much like we do in our research, Du and colleagues propose a system for multi-user exploration of a BIM model (Du et al., 2016). The BIM model of a nearly finished office building is created in Autodesk Revit, then exported into Unity3D, a popular game engine, and with an intermediate material adjustment step performed in 3DS Max; therefore, no real-time modifications of the model are possible. The multi-user functionality is achieved with the use of Photon Networking plugin for Unity 3D. The movement of users is implemented in a walk-through mode, where the first-person virtual camera of each user moves on a pre-defined trajectory following keyboard input of the user, while natural user movements are limited to looking around. This system is further developed in a follow-up publication where a possibility to introduce real-time changes to the BIM model is introduced (Du et al., 2018). When a user changes the BIM representation in the Revit environment, the change is published on the server and communicated to other users in the VR environment. Such tested changes included modifying the size and positions of objects such as chairs and columns, with their initial and final states being synchronized to VR users. A pilot study conducted by the authors showed that the collaborative system encouraged communication between stakeholders and facilitated the creation of a shared vision of the project.

Kieferle and Woessner presented a workflow enabling bidirectional data exchange between Revit and COVISE, VR software (Kieferle,2015). The data exchange is implemented with two specialized plugins using TCP sockets. Users can change a range of exposed parameters. The framework was tested in a CAVE VR system that naturally supports collaboration since its users are collocated and visible to each other. However, the system supports other rendering clients such as phones and tablets and can be used by a team even in a remote meeting session. While the system presents a big step forwards in integrating BIM and VR, authors note the difficulty involved in the development of the system and limitations posed by inaccessible parts of the involved APIs.

More recent publications discuss the possibilities of combining parametric design in BIM and VR visualization. The work of Moubile presents a system where parametric modelling is enabled by Grasshopper and Karamba plugins for Rhino and Unity3D rendering engine is used to create a virtual scene (Moubile,2018). The link between BIM and VR is established directly in a custom Grasshopper component, with the 3D data being serialized in the JSON format and forwarded to Unity3D VR client. In the virtual environment, a user can select structural elements with controllers and see the corresponding structural information displayed. In addition, certain elements can be removed from the structural calculation. The interaction is accomplished via a menu where a user can change the values of loads and grid dimensions. The integration of structural information into the virtual environment leads to better understanding of the developed design already at an early stage.

Similarly, Coppens and colleagues propose a system where a parametric model created with a Grasshopper script in Rhino is visualized in VR (Coppens et al., 2018). Variables defining the outcome of the calculation are exposed to the user immersed in VR as input sliders. The system consists of a plugin that connects a Grasshopper script to a web-socket, a web server and a client application in which a virtual environment is created. The same authors discuss the possibilities of extending their system for multi-user scenarios in a later publication (Coppens and Mens,2018), focusing on the description of demands and challenges. However, no multi-user prototype is presented.

Quinn and colleagues present another solution for streaming 3D geometry from parametric modelling software to VR clients (Quinn et al., 2018). In their work, a user can interact with structures defined in Grasshopper with the help of the Kangaroo plugin, such as trusses, and bridges etc. Mesh vertices are streamed directly from Grasshopper to Unity3D, either with gHowl plugin for Grasshopping working over User Datagram Protocol (UDP) or by using a NodeJS server and ZeroFormatter library for serialization and deserialization of data. The user can pull and push elements of the structure with controller-enabled natural movements and see the changes in tension forces displayed with colour gradients. Horikawa suggests an alternative method of connecting Rhinoceros with its 
parametric modelling functionality in Grasshopper and Unity3D (Horikawa,2019). In his solution, Rhino runs in its experimental headless server form, Rhino Compute (McNeel and Associates, 2019) that is accessed from Unity $3 D$ at runtime via Rest API.

The appreciation of the combined power of BIM and VR has lead to appearance of commercial solutions as well. Enscape is a plugin for Revit and Rhino that provides a VR viewer for walkthrough explorations of designs, with changes made in Revit being instantaneously displayed in VR (Enscape,2019). Enscape has been used in several published case studies such as an examination of the bill of quantities in a furnished house (Davidson et al., 2020) and a lifecycle cost assessment of building facades (Kamari et al., 2021). Mindesk plugin for Rhinoceros exposes Rhino modelling functionality to $\mathrm{VR}$, allowing a user to use controllers and a VR version of its user interface to create designs in an immersive mode, and including an option to use Grasshopper for parametric modeling (Mindesk,2019b). A multi-user option is available as well, allowing up to four users to work on designs collaboratively (Mindesk,2019a). The networking setup includes one admin user whose workstation runs Rhino with Mindesk Link software and keeps the 3D model that can be edited and saved, while other connecting users only get access to the $3 \mathrm{D}$ model during the live session. Fuzor is a collaborative tool that supports bidirectional links with Revit and Rhino among other software solutions, allowing users to explore and edit models in VR (Kalloc Studios, 2018). Collaborating users are represented by photorealistic humanoid avatars that are controlled either by keyboard input or head-mounted display (HMD) tracking. Zaker and Coloma used Fuzor in a case study evaluating the use of VR in a real-world BIM-based project, with pairs of co-workers performing collaborative clash-detection in a complex pipework system (Zaker and Coloma, 2018).

Unlike commercial BIM + VR solutions with powerful functionality and very complex sets of tools, BIMFlexi-VR is not aimed at from-scratch modeling, but at accessible collaborative reviewing of designs in the early planning stage. The system is designed to equip users with means of quickly changing properties of the construction without overwhelming them with complex functionality. To allow maximum flexibility in user representations, interaction design and navigation capabilities, BIMFLexi-VR is implemented in a game engine rather than as a specialized plugin for an authoring tool. The presented solution is built on the concept of coupling Rhino Compute with Unity3D suggested by Hirokawa (Horikawa,2019).

\section{BIMFLEXI-VR FRAMEWORK}

\subsection{BIMFlexi Workflow Vs Traditional Design Process}

Industrial building designs demonstrated in this paper are based on a pilot project of a typical food and hygiene production facility located in Austria. As the company is growing quickly, it is confronted with challenges of expanding production processes, and changing equipment every one or 2 years. When a change in

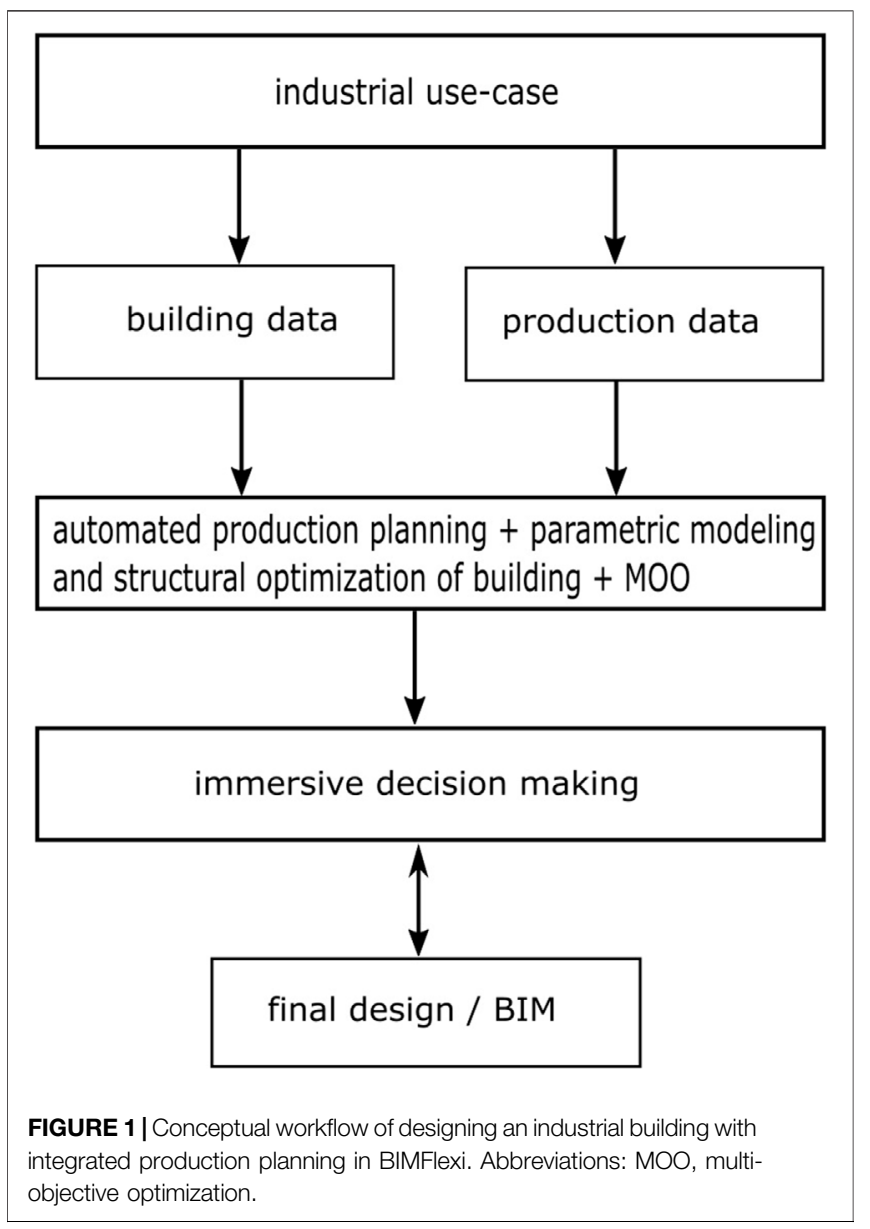

production occurs, spatial restrictions of the production facility force the production owners to expand or rebuild the existing building.

In the typical planning process in industrial building design, a production planner presents one layout of the desired production plan to an architect, and without possible future changes in production being taken into account. The architect then manually designs a building around this exact production layout. After the completion of the architectural design, a structural engineer performs calculations to prove its structural integrity. The initial design will likely need modifications, resulting in iterations of it being passed between the production planner, the architect and the structural engineer, and while planning decisions also need to meet the approval of the production owner.

In the BIMFlexi workflow illustrated in Figure 1, these repetitive iterations are avoided by taking into account all stakeholder needs simultaneously already in the early stages of the planning process. Work on a particular industrial use-case starts with the analysis of the production needs and geometric criteria for the future building. At this step, input from all stakeholders is gathered. In contrast to the traditional planning process, several production options are considered at this stage to assure flexibility of the future building. The assembled building and production data are used as input in 


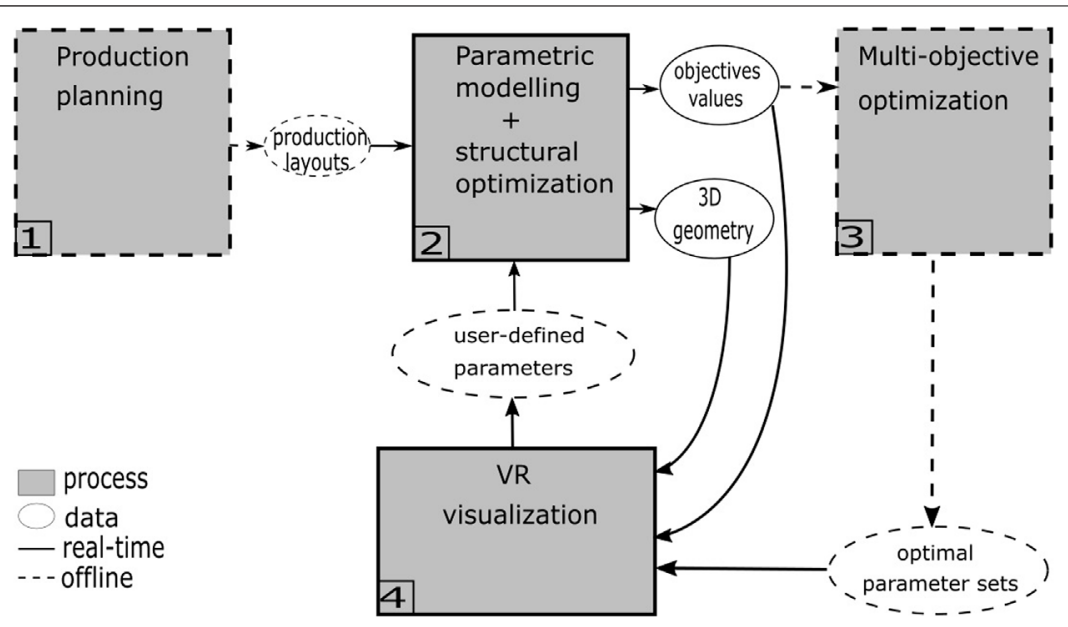

FIGURE 2 | Automated processes and connections between them in BIMFlexi. Abbreviations: MOO, Multi-objective optimization.

the innovative automated step of BIMFlexi-generation of production layouts and algorithmic creation and assessment of the building structure from sets of input parameters. This automated step includes further performance assessment of the resulting building structures based on their environmental and economic impact in the process of multi-objective optimization. The results of automated modeling and optimization are collaboratively inspected in immersive VR by all stakeholders, where they are able to adjust different relevant structure parameters and see corresponding changes in the building geometry and resulting performance metrics. Through this process, stakeholders arrive at the consensus on the building properties, resulting in the final BIM design.

Figure 2 illustrates the detailed implementation of the automated part of this conceptual workflow that is directly connected to the main contribution of this paper, the BIMFlexi-VR framework. The automated design of a flexible industrial building contains offline and online steps. Step 1, Production planning, is conducted prior to the real-time part of the workflow and results in the data output describing production layouts $^{1}$ (the specifics of input and output files relevant to BIMFlexi-VR are described further). Production layouts serve as input, along with parameters defining the structure of the building, to Step 2, Real-time parametric modelling and structural optimization process.

The parametric modelling and structural optimization process results in 3D geometry of the building and a set of metrics, which we further refer to as objectives, and that are used to assess the quality of the construction. The objectives are used in the further offline Step 3, Multi-objective optimization (MOO). During $\mathrm{MOO}$, sets of structural parameters which result in the best construction outcomes are found. To do this, the parametric

${ }^{1}$ Production layouts are generated and optimized automatically, the description of this automatic process can be found in our previous publication Reisinger et al. (2021b). modelling and structural optimization process of Step 2 is run multiple times, each time with a different combination of structure-defining input parameters. The resulting objectives are used to rank each specific combination of input parameters against other combinations. Several best-ranked parameter sets are saved as the output of MOO. Step 4, Realtime VR visualization of the construction is coupled directly to the parametric modelling and structural analysis process. In VR, users collaboratively change structural parameters of the future construction; these user-defined parameter values are then used in the parametric modelling and structural analysis process, with the resulting 3D geometry and values of objectives being sent back into the immersive visualization process. The optimal parameter sets obtained in the MOO process of Step 3 are also made available to the VR visualization process as input; users can switch between these recommended parameter sets and see the real-time constructions created by the parametric modelling and structural analysis process along with the corresponding values of objectives. Research associated with the development of the specific objectives used in BIMFlexi and methods of MOO resulting in optimal parameter sets is currently in the process of publication.

The advantage of our solution is that it provides users with full flexibility of parameter choice while also recommending optimal solutions. The detailed demonstration of user possibilities around parameter selection follows in Section 3.2, which describes BIMFlexi-VR, our real-time framework implementing collaborative VR visualization and its bidirectional real-time connection to the parametric modelling and structural analysis process.

\subsection{BIMFlexi-VR-Architecture and Usage}

The BIMFlexi-VR framework is based on the real-time link between the parametric modeling and structural optimization core implemented in Grasshopper for Rhino and an immersive collaborative virtual environment developed in Unity3D game engine. The foundation of this link is RhinoCompute, an 


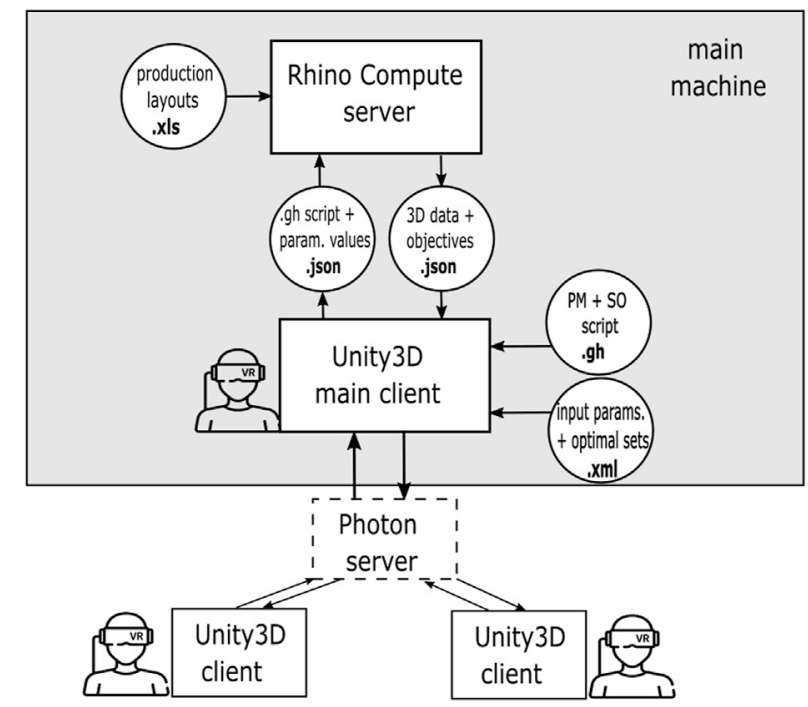

FIGURE 3 | BIMFlexi-VR framework. Abbreviations: PM, parametric modelling; SO, structural optimization.

experimental headless implementation of Rhino that can be accessed by external processes via REST API. A diagram illustrating the work of BIMFlexi-VR is presented in Figure 3. In the BIMFlexi-VR workflow, RhinoCompute is run in the background and available at a specific port on the main Windows machine with a licenced installation of Rhino 7 and several plugins for Grasshopper that are needed in the structural optimization framework. The main Unity3D client runs on the same machine, where several files necessary for the correct performance of the parametric modelling and structural optimization core and the communication between Unity3D and RhinoCompute are also saved.

At the startup, the main Unity3D client reads and processes two input files. The first file contains Grasshopper script defining the parametric modeling and structural optimization algorithms used in the project. After being loaded into Unity3D, this script is serialized into. JSON format and sent to the RhinoCompute server as a REST request. RhinoCompute performs calculations defined within this Grasshopper script and sends the resulting $3 \mathrm{D}$ geometry as REST response back to the main Unity3D client. This request-response communication is repeated every time a user chooses to recalculate the structural model after modifying its parameters in VR. The multi-user functionality is implemented in Unity3D with Photon Networking for Unity (PUN) plugin. After the main client has received $3 \mathrm{D}$ geometry from RhinoCompute, it is interpreted as mesh data in the internal Unity3D description and forwarded to other Unity3D clients. Alongside the Grasshopper script defining structural calculations, the main Unity3D client loads an. $x m l$ file where possible values of structural parameters which can be modified by users are defined. Further sections describe in detail how these values are set by users in VR and subsequently used in the calculations performed by RhinoCompute. The optimal sets of structural parameters

\section{Layout Selection}

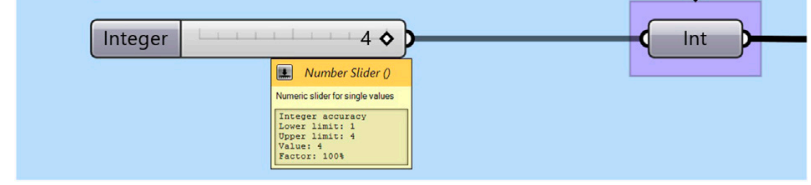

FIGURE 4 | User input is implemented with sliders in the usual Grasshopper workflow, and with annotated auxiliary variables in our workflow using RhinoCompute. input. xml file.

obtained at Step 3 of the BIMFlexi workflow, Multi-objective optimization, and are included in the input. xml file as well. Production layouts calculated as the output of Step 1 of the BIMFlexi workflow, Production planning, are saved in the format and used as input in the Grasshopper script performing structural calculations with the help of NPOI-Excel plugin for Grasshopper.

In the current implementation of BIMFlexi-VR, the Rhino Compute server and the main Unity3D client must run on the same machine since Rhino Compute is accessible at localhost. The Photon server implicitly used by PUN for within-Unity distribution can be run either in its standalone version on the same computer, or any computer accessible through the local network, or in the cloud. This way, BIMFlexi-VR allows both collocated and distributed user collaborations.

\subsubsection{User Input}

In parametric modeling with Grasshopper, user input is enabled through sliders that can be directly manipulated by users within the script. Once a user has changed the value of the slider, the parametric model is automatically recalculated, and the result is displayed in the Rhino editor. Figure 4 illustrates an integer 


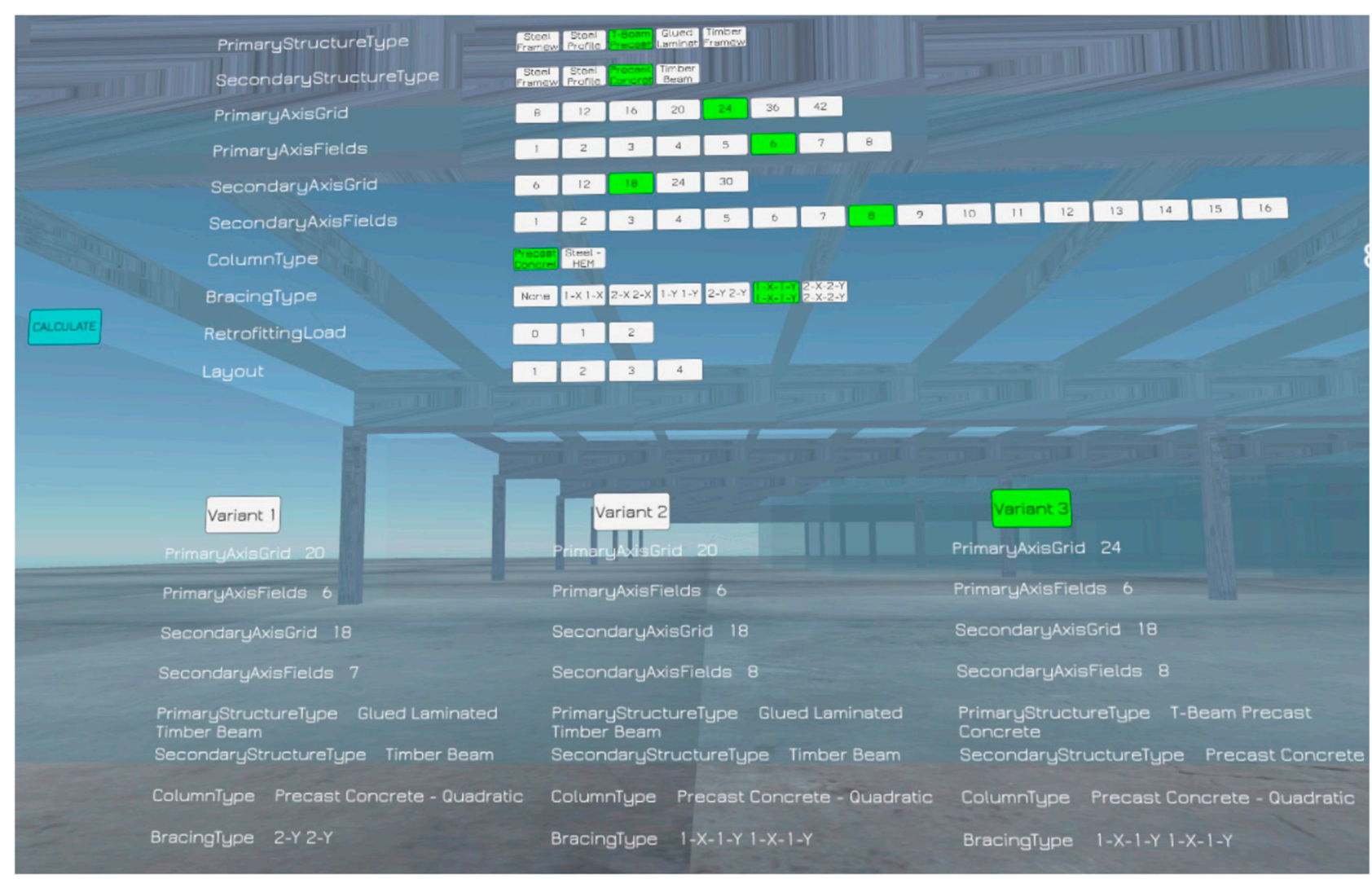

FIGURE 6 | In-game menu allowing users to choose parameters of the structural system and the preferred production layout (screenshot of a user's VR view). Variant 1, Variant 2, and Variant 3 correspond to optimal parameter sets found during multi-objective optimization. Parameters displayed in green belong to the current selection.

accuracy slider from the parametric modeling and structural optimization script that allows a user to choose one of four production layouts considered in the structural calculation. However, real-time user input is handled differently when the Grasshopper script is executed on RhinoCompute as a part of the immersive BIMFlexi-VR workflow.

For a parameter to receive values assigned by user input, an auxiliary variable is introduced in the Grasshopper script after the slider and annotated with RH_IN (also illustrated on the example of layout choice in Figure 4). When the Grasshopper script sent by Unity3D to RhinoCompute in a. JSON file is de-serialized, all variables marked with $\mathrm{RH}$ _IN are interpreted as requiring user input.

The values of all Grasshopper parameters marked with RH_IN as those requiring user input must be contained in the JSON request created in Unity3D. All such user-defined input parameters and their possible values are described in an xml file that is loaded by the main Unity3D client at startup. Figure $\mathbf{5}$ demonstrates an. $\mathrm{xml}$ entry for the input parameter defining the production layout (the same parameter as in Figure 4). The name of the parameter must coincide to its annotation in the Grasshopper script after the prefix RH_IN. A parameter can have either a discrete set of values which are listed in the entry or a range of values, in which case minimum and maximum values are defined instead. The default value of the parameter is set in the field currentValue. The xml description does not contain the type of the parameter, it is inferred from the type of the annotated variable in the Grasshopper script. Alongside the list of parameters, the input. xml file contains the description of optimal parameter combinations that were identified at Step 3, Multi-objective optimization. The parameters and their possible values (along with sets of optimal parameters) are exposed to users in the form of a menu placed within the virtual scene that can be seen in Figure 6. Users are able to choose different parameter values via virtual cursor interaction, either setting individual parameters separately or selecting an optimal set of values.

\subsubsection{Calculation Output}

Elements of the Grasshopper script that are created during the computation and should be sent to Unity3D as a part of the REST response are annotated with the prefix RH_OUT (illustrated in Figure 7). These elements correspond to $3 \mathrm{D}$ meshes calculated by RhinoCompute and floating-point values of objectives. The output meshes are interpreted as Rhino meshes defined in $3 d m I O$ library included into the Unity3D project, converted to Unity3D mesh representation and constructed at runtime. Examples of the building construction generated in BIMFlexiVR can be seen in Figure 8. 

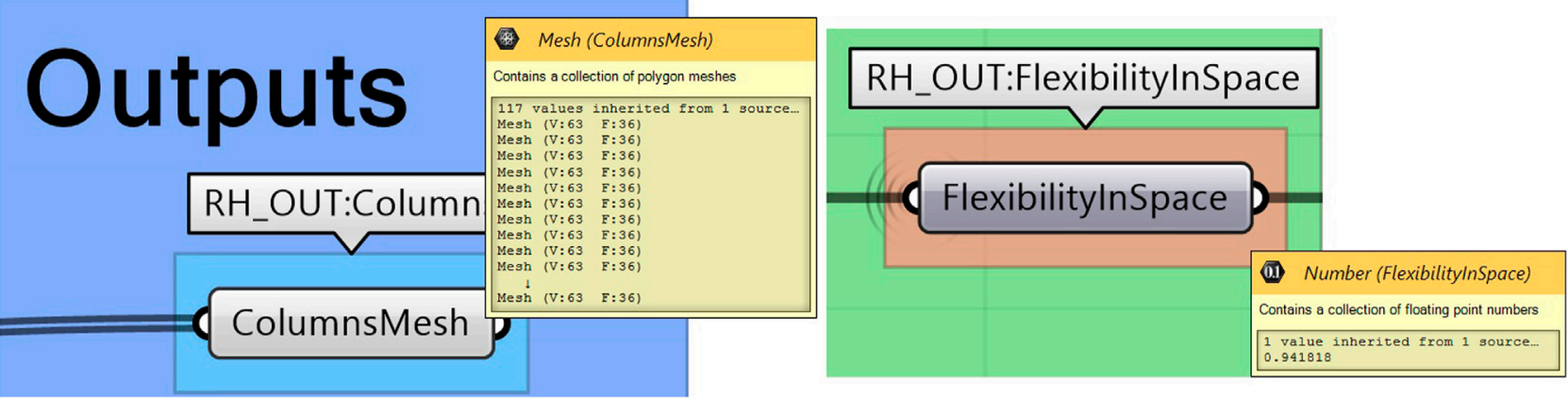

FIGURE 7 | Annotations marking an element of a Grasshopper script as an output to be sent as REST response. Left: an output element of type Mesh; right: an output element of type Number (float).

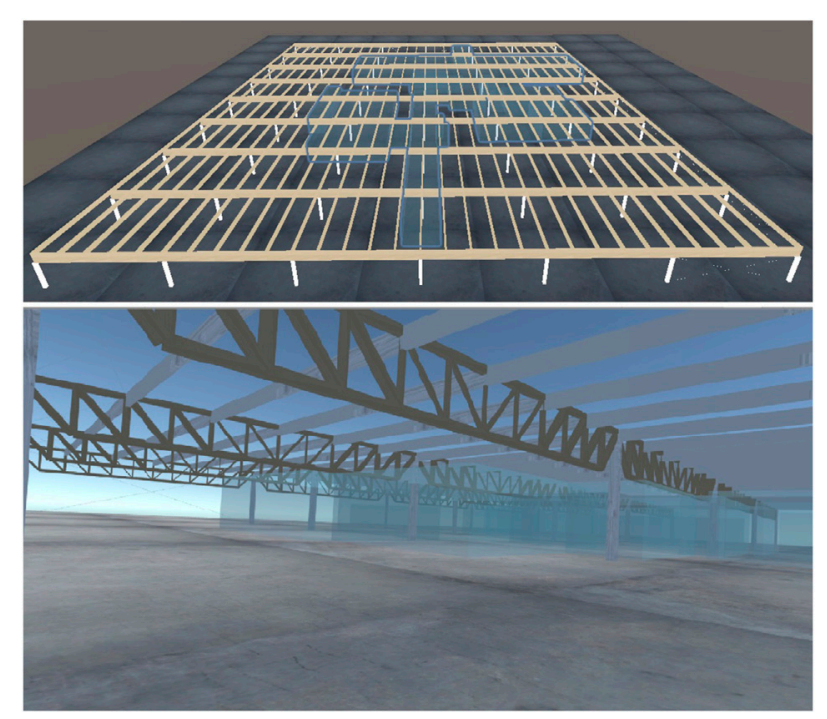

FIGURE 8 | Examples of parametrically-generated industrial buildings in BIMFlexi-VR. Top: top-down view in the Unity3D editor; bottom: a screenshot from the point of view of an immersed user. Semi-transparent elements depict production layouts - areas that would be occupied by the production line.

Values of the objectives corresponding to the current choice of the structural parameters are displayed to users on two radar charts (depicted in Figure 9), one summarizing the objectives related to costs and environmental impact and the other one presenting the objectives describing the flexibility of the construction in respect to future changes in production. The values of the objectives are presented in percent of their fulfilment. Specifically, if the value of the objective is $100 \%$, the current structural layout is optimal in terms of usability criteria described by this objective.

\subsubsection{Multi-User Workflow}

The multi-user functionality of BIMFlexi VR is build with PUNcontained mechanisms for state synchronization and remote procedure calls (RPCs). State synchronization is used to maintain the positions of users updated across the network. User interactions with the content and the synchronization of the results of RhinoCompute calculations are implemented with RPCs following the conceptual diagram presented in Figure 10. By interacting with the in-world menu, each user can either change the value of a structural parameter or request a calculation. Both these actions result in an RPC call to the main Unity3D client. If the RPC contains a parameter change, this change is synchronized to all connected clients. If it is a calculation request, a new request-response exchange with RhinoCompute is initiated by the main Unity3D client. The resulting meshes are interpreted by the main client, and RPC calls containing a command to create a mesh with a defined set of vertices (extracted from the Rhino mesh definition) and triangles are sent to all connected clients.

\subsubsection{Hardware}

The current version of BIMFlexi-VR supports Oculus Quest (v.1 and v.2) as head-mounted displays. The main client has been tested on a Windows PC with Intel Core i7-9750H processor with 6 cores and Nvidia GeForce RTX 2070, with Oculus Quest v.2 connected wirelessly over AirLink. Other Unity3D client can run either on Windows, with Oculus Quest connected with Oculus Link/AirLink, or as standalone Android applications build directly onto Oculus Quest.

\section{PILOT EVALUATION}

A pilot evaluation study aimed at comparing desktop-based and immersive collaboration processes within the conceptual workflow of BIMFlexi has been designed and conducted. The main goal of this pilot evaluation was to identify the advantages and limitations that a collaborative immersive VR environment of BIMFlexi-VR brings into the overall automated industrial building design process developed in the BIMFlexi project.

In the pilot experiment, three multidisciplinary teams of users worked together on two tasks: 1) conducting a variant study of three different structural parameter sets in order to select the best fitting one for a given production layout, and 2) designing an industrial building structure that would accommodate a given 


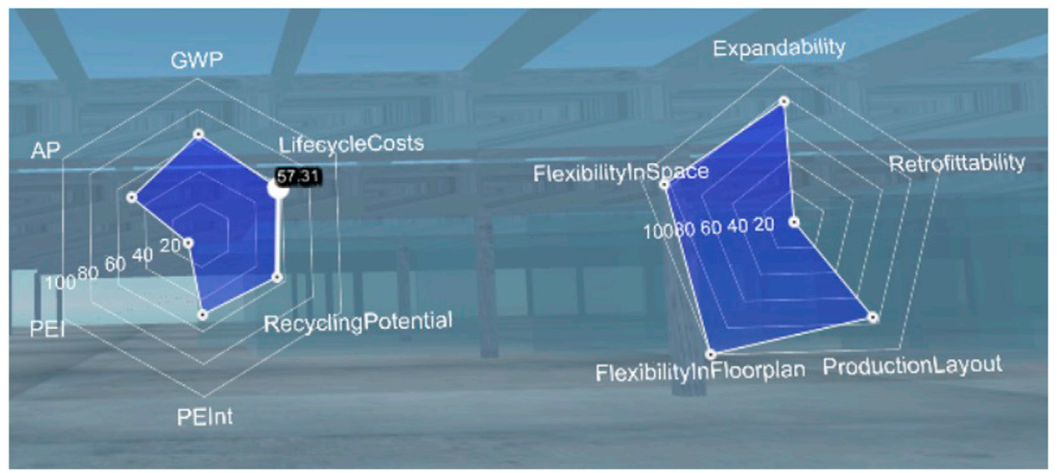

FIGURE 9 | Radar charts displaying the values of the objectives achieved with the current parameter selection (a screenshot of the immersed user's view). The left chart shows the objectives describing the costs and environmental impact, the right one those describing the flexibility of the building. Abbreviations: GWP, global warming potential; AP, acidification potential; PEl, primary energy use; PEInt, non-renewable primary energy use.

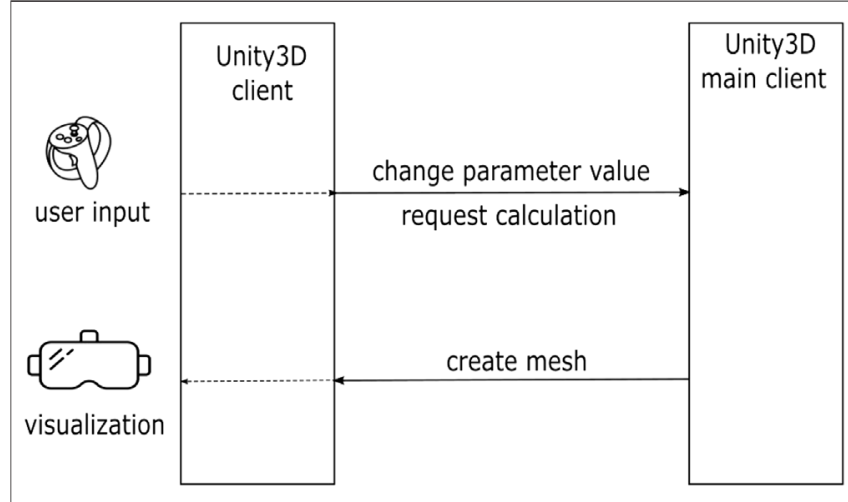

FIGURE 10 | RPC exchanges between the main Unity3D client and all other clients in BIMFlexi-VR.

production layout in a way maximizing the flexibility of the structure. Each team consisted of an architect and a structural engineer. Each team worked with two types of setup-1) the parametric modeling and structural optimization Grasshopper script viewed directly in the Rhinoceros editor on desktop, with collaboration being enabled by screen sharing, and 2) in immersive VR, within the BIMFlexi-VR framework. Team members shared the same office and were able to talk to each other without any mediating technology in both cases.

\subsection{Evaluation Metrics}

A subjective questionnaire was used to assess the overall acceptance of the technology. The questionnaire addressed the ease of use of each of the setups, its potential in terms of learning and information transfer, collaborative aspects and decision making support. The questions used for each category are summarized in Table 1. The answers were recorded on a Likert-style 5-point scale. In addition, the time each team took to accomplish both tasks in the two evaluation settings was recorded, as an objective metric of the efficiency of each interface. A de-briefing session took place after the evaluation, in which user experiences with the tested setups were discussed in detail and informal feedback was collected.

\subsection{Setup and Procedure}

Both phases of the evaluation took place in a university office, with two participants from a team, and two experimenters present. At the beginning of the evaluation, the experimenters introduced the BIMFlexi project and explained two experimental workflows - the desktop-based one, where participants manipulate parameters directly in Grasshopper and the immersive one-which are both novel in industrial building design. The main tasks - the variant study and the design of an industrial structure-were introduced. Modes of interaction in each experimental setup were demonstrated immediately before the evaluation of that setup began. Performing the tasks in each experimental setup was directly followed by filling in the questionnaire, while the de-briefing session, where both setups were discussed, and took place in the end of the evaluation.

During the part of the evaluation where participants worked with the parametric modelling script in Grasshopper and Rhino editor, each of two participants from a team had their own computer with a copy of the parametric script. The desktop of one of the participants was shared on a big screen, allowing both users to have a shared view. For the variant study, both participants received print-outs defining the structural parameters of three building layouts to be compared. In addition, layouts were summarized inside the Grasshopper script as text panels. To achieve a configuration corresponding to each of three layouts, participants had to manipulate individual parameter sliders. The sections of the Grasshopper script demonstrating layout descriptions and input sliders are presented in Figure 11. In the immersive part of the evaluation, three structural layout variants were summarized in the user menu as parameter sets; users were able to switch from one parameter set to another with one press of the virtual button. In the version of BIMFlexi-VR as the time of the evaluation, the calculation of the construction started automatically when any of the menu buttons was pressed. 
TABLE 1 | The questionnaire used in our pilot evaluation.

\begin{tabular}{|c|c|c|}
\hline Category & Code & Question \\
\hline \multirow[t]{4}{*}{ Ease of use } & E1 & How easy was it to switch between different variants? \\
\hline & E2 & How easy was it to do variant studies? \\
\hline & E3 & How intuitive was the interface? \\
\hline & E4 & How often did you ask for help? \\
\hline \multirow[t]{4}{*}{ Learning } & L1 & How well did you understand the structure of the building? \\
\hline & L2 & How well did you understand the spatial layout of the building? \\
\hline & L3 & How well could you understand the interaction between production and building? \\
\hline & Ł4 & How easy was it to understand the differences between different variants? \\
\hline \multirow[t]{3}{*}{ Collaboration } & C1 & How easy was it to share/demonstrate your point of view to your team partner? \\
\hline & $\mathrm{C} 2$ & How easy was it to understand the perspective of your team partner? \\
\hline & C3 & How well could you collaborate and support your team partner? \\
\hline Decision making & D1 & How helpful was the tool in interdisciplinary decision making? \\
\hline
\end{tabular}
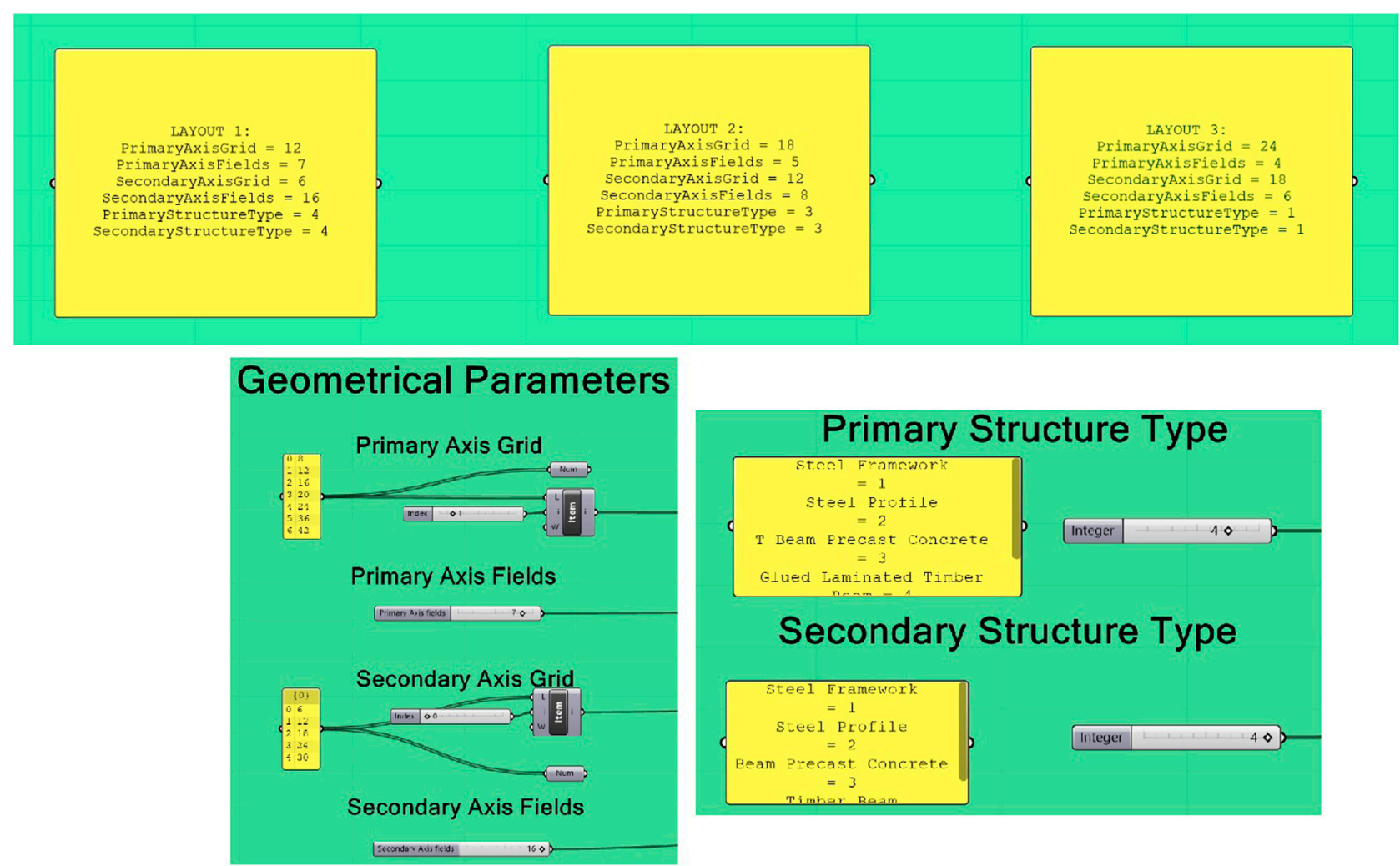

FIGURE 11 | Instructions embedded into the Grasshopper script and slider-based interaction during the desktop-based part of the evaluation.

The variant study and the free design phase were conducted during the same session both on desktop while working with Grasshopper and in VR. The transition from the variant study to the free design phase was noted by the experimenter based on the feedback from the participants. The free design task was finished when both participants from a team agreed that the design was final and indicated it to the experimenters. There was no time limit set for the completion of the tasks, neither were the teams encouraged to finish them as quickly as possible. Participants freely commented on their actions as they were performing the tasks and talked to each other and the experimenters during all parts of the evaluation.

\subsection{Results}

\subsubsection{Metrics}

The mean questionnaire scores are summarized in bar charts presented in Figure 12. Taking into consideration the exploratory nature of our evaluation and a relatively low number of 


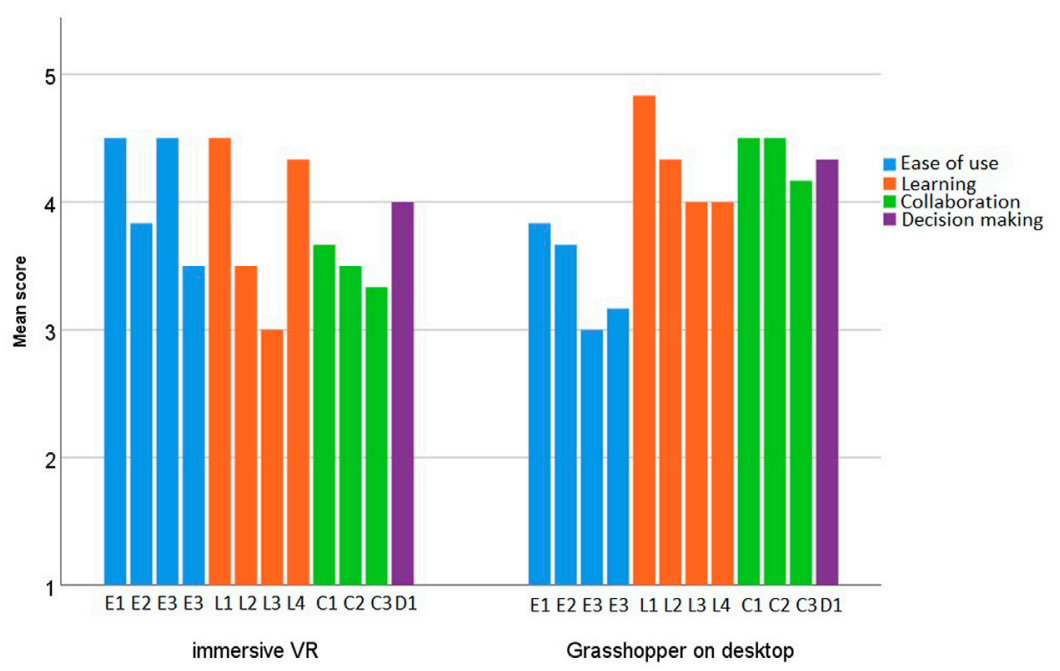

FIGURE 12 | Bar charts of questionnaire scores. The letter codes correspond to question codes in Table 1.

TABLE 2 | Task completion times. Abbreviations: GH = Grasshopper.

\begin{tabular}{lccc}
\hline & Team & Variant study, mins & Free design, mins \\
\hline GH + Rhino & 1 & 25 & 6 \\
& 2 & 20 & 10 \\
& 3 & 19 & 6 \\
\hline BIMFlexi-VR & 1 & 6 & 7 \\
& 2 & 8 & 20 \\
& 3 & 10 & 10
\end{tabular}

participants, especially if we were to consider different participant roles (architect and structural engineer), we did not perform formal statistical tests.

Both setups tested in the evaluation were positively received by the participants. BIMFlexi-VR received tentatively higher scores associated with the ease of use than the Grasshopper + Rhino setup (bar charts depicted in blue in Figure 12). Indeed, the perceived ease of use is reflected in the time results as well, especially in the variant study part the evaluation (see Table 2). Scores associated with learning and skills transfer were mixed (the orange colour-coding), with the understanding of interaction between the production and the structure tending to have higher score in the desktop setup than in VR, but the differences between various types of construction being better visible in VR. To our surprise, the collaborative aspects (depicted in green) received slightly higher scores in the desktop setup. Our observations and participants' feedback further expand on these results.

\subsubsection{Observations and User Feedback}

As indicated in the resulting task times, the variant study took longer to accomplish on desktop while working directly in Grasshopper because participants had to change every slider value individually. When a slider is moved in Grasshopper, the construction is automatically recalculated, the calculation taking several seconds, and the editor being unresponsive until it is finished. Therefore, it was impossible for participants to move sliders quickly one after another, the workflow being slower than preferred. In BIMFlexi-VR, although users have to wait for the result of the calculation several seconds as well (the calculation being performed by the same Grasshopper script), the delay impacts them less since the calculation happens in the background while the VR environment itself responds normally. Since the calculation takes several seconds, participants expressed the wish to re-calculate the construction on request and not every time when a parameter value has changed, both in Grasshopper directly and in BIMFlexi-VR. We incorporated this feedback into BIMFlexi-VR, while it is not possible to change this behaviour in Grasshopper + Rhino workflow.

Participants did not introduce many changes into their constructions during the free design phase on desktop, since trying out more layouts involved more interactions with sliders. In BIMFlexi-VR, on the contrary, participants tended to try out more parameter combinations during the free design task. While doing that, participants experimented with parameter values, and wanting to see even a-priori unreasonable combinations visualized.

In Team 1, participants were guided primarily by the height of the resulting construction, the costs of the building and the flexibility of its floorplan displayed as objectives. Materials used in the construction were important for decision making for both participants from the team; the structural engineer additionally emphasised the role of load-bearing structures, while the architect focused on the geometric properties such as axis grids. In Team 3, participants took into account objectives reflecting the flexibility of the building and the appearance of the construction, also focusing on the materials (for example, concrete was the least preferred option). Participants from Team 2 concluded that Grasshopper + Rhino setup was more suitable for the engineering part since it allowed to see the ways the calculations were performed directly in the script and 
provided more control for the structural engineer. The immersive setup was judged as visually better and easier to interact with, being ideal for presentations, especially for building owners.

All teams agreed that while seeing the building from the inside gave a good impression of the construction and was important for the understanding of the future building, the large scale of the construction made it difficult to see where the hall ended from the inside of the building. A top-down view of the construction on the smaller scale was suggested as a beneficial addition that would enable users to see everything at once. Finally, users wished to see the selection cursor of the team partner, so that users could point at the parameters in the menu while discussing them. We incorporated this feedback.

\subsection{Discussion}

The pilot evaluation, although conducted with only three teams, allowed us to identify important advantages and requirements of collaborative work in immersive VR. Our experimental sessions provided a robust indication that BIMFlexi-VR enables users to switch between different parameter values efficiently, allowing them to view multiple possible designs in a short time. The power of immersive VR in terms of creating a compelling visualization was confirmed by participants as well. We have seen that our framework encourages free experimentation, indicating that BIMFlexi-VR could be useful not only in the AEC industry as such, but in architecture and engineering education as well.

All participants indicated that an in-depth understanding of the examined construction requires not only a visualization that is scaled 1:1 like a real building, but also a holistic scaled-down view of the whole construction. The possibility to see the $3 \mathrm{D}$ model of the parametrically designed building in the Rhino editor was cited as a major advantage of the desktop-based workflow. We are investigating an approach of creating a tabletop-sized replica of the parametric model within the immersive environment of the real-world scaled model. Alternatively, an algorithm allowing to switch between different model sizes during the immersive workflow-first observing the virtual building on a tabletop and later on switching to the full-scale representation-could be introduced.

We saw indications that different user roles in an interdisciplinary team might require different sets of tools and interaction possibilities. For example, while a simple visualization might be sufficient for an uninvolved observer, a structural engineer would require the possibility to control more precise aspects of the calculation. Although production planners were not involved into the pilot evaluation, it is probable that showing specific machine lines, and production arrangements would be beneficial for their understanding of the construction. BIMFlexiVR provides a solid technical foundation for collaborative work in industrial building design; the challenge of its integration into day-to-day design practices is in identifying interactions and workflows meaningful for multidisciplinary teams. Identifying control parameters and interactions that are specific to each user role and including them into BIMFlexi-VR-enabled multidisciplinary workflow is part of our future work on the project.

\section{CONCLUSION}

In this paper, we presented BIMFlexi-VR - our immersive framework for collaborative design of industrial buildings that is a part or the larger project BIMFlexi. BIMFlexi is aimed at bringing together interdisciplinary teams, especially linking production planning and structural design in the early work stages, in order to design flexible, environmentally friendly, and long-lasting industrial constructions.

Early testing showed that BIMFlexi-VR was positively received by users, allowed to conduct variant studies of structural constructions quickly and to experiment with structural parameters in an unconstrained and creative way. We have indicated directions of future work, primarily focusing on the development of suitable multidisciplinary workflows within our immersive framework, alongside with improving user capabilities in terms of interaction and acquiring a holistic impression of the building. We believe that the intersection of parametric BIM and immersive multi-user $\mathrm{VR}$ is placed ideally to meet the requirements of multidisciplinary collaboration in AEC industry, streamlining communication and enabling faster, better, and more sustainable designs.

\section{DATA AVAILABILITY STATEMENT}

The raw data supporting the conclusion of this article will be made available by the authors, without undue reservation.

\section{ETHICS STATEMENT}

Ethical review and approval was not required for the study on human participants in accordance with the local legislation and institutional requirements. The patients/participants provided their written informed consent to participate in this study.

\section{AUTHOR CONTRIBUTIONS}

IP implemented the BIMFlexi-VR framework, co-designed the pilot evalution, and wrote the manscript draft; JR co-designed the pilot evaluation, co-designed interactions in BIMFlexi-VR, and contributed to the manuscript; HK co-designed the BIMFlexi-VR framework and contributed to the manuscript. IK co-designed the BIMFlexi-VR framework and contributed to the manuscript.

\section{ACKNOWLEDGMENTS}

The authors would like to acknowledge the support by the Austrian funding institution FFG (Österreichische Forschungsförderungsgesellschaft) for the research project "BIMFlexi" within the FFG BRIDGE program (30th call for tenders, Grant No. 877159). Open access publishing is funded by the TU Wien library. 


\section{REFERENCES}

Anderson, A., Dossick, C., Azari, R., Taylor, J., Hartmann, T., and Mahalingham, A. (2014). Exploring Bims as Avatars: Using 3d Virtual Worlds to Improve Collaboration with Models. ASCE Construction Res. Congress. 10, 179-188. doi:10.1061/9780784413517.019

Anderson, A., Sturts, C., Dossick, J., Iorio, J., and Taylor, E. (2017). The Impact of Avatars, Social Norms and Copresence on the Collaboration Effectiveness of Aec Virtual Teams. J. Inf. Technology Construction (Itcon). 22, 287-304. Available at http://www.itcon.org/2017/15.

Boton, C. (2018). Supporting Constructability Analysis Meetings with Immersive Virtual Reality-Based Collaborative Bim 4d Simulation. Automation in Construction. 96, 1-15. doi:10.1016/j.autcon.2018.08.020

Bouchlaghem, D., Shang, H., Whyte, J., and Ganah, A. (2005). Visualisation in Architecture, Engineering and Construction (Aec). Automation in Construction. 14, 287-295. doi:10.1016/j.autcon.2004.08.012

Coppens, A., Mens, T., and Gallas, M.-A. (2018). Parametric Modelling within Immersive Environments - Building a Bridge between Existing Tools and Virtual Reality Headsets.. Hangzhou, China: Springer International Publishing

Coppens, A., and Mens, T. (2018). "Towards Collaborative Immersive Environments for Parametric Modelling," in Cooperative Design, Visualization, and Engineering. Editor Y. Luo (Cham: Springer International Publishing), 304-307. doi:10.1007/978-3-030-00560-3_44

Davidson, J., Fowler, J., Pantazis, C., Sannino, M., Walker, J., Sheikhkhoshkar, M., et al. (2020). Integration of $\mathrm{Vr}$ with Bim to Facilitate Real-Time Creation of Bill of Quantities during the Design Phase: a Proof of Concept Study. Front. Eng. Management. 7, 396-403. doi:10.1007/s42524-019-0039-y

Du, J., Shi, Y., Mei, C., Quarles, J., and Yan, W. (2016). "Communication by Interaction: A Multiplayer Vr Environment for Building Walkthroughs," in Proceedings of the Construction Research Congress 2016, 2281-2290. doi:10. 1061/9780784479827.227

Du, J., Zou, Z., Shi, Y., and Zhao, D. (2018). Zero Latency: Real-Time Synchronization of Bim Data in Virtual Reality for Collaborative Decision-Making. Automation in Construction. 85, 51-64. doi:10.1016/j.autcon.2017.10.009

[Dataset] Enscape, G. (2019). Enscape - Archtectural Virtual Reality.

Fernandes, K. J., Raja, V., White, A., and Tsinopoulos, C.-D. (2006). Adoption of Virtual Reality within Construction Processes: a Factor Analysis Approach. Technovation. 26, 111-120. doi:10.1016/j.technovation.2004.07.013

[Dataset] Horikawa, J. (2019). Rhino Compute with unity3d.

Johansson, M., Roupé, M., and Viklund Tallgren, M. (2014). "From Bim to VrIntegrating Immersive Visualizations in the Current Design Process," in Fusion-Proceedings of the 32nd eCAADe Conference, 261-269.

[Dataset] Kalloc Studios, F. (2018). Fuzor - Vr Collaboration.

Kamari, A., Paari, A., and Torvund, H. (2021). Bim-enabled Virtual Reality (Vr) for Sustainability Life Cycle and Cost Assessment. Sustainability. 13, 1-24. doi:10. 3390/su13010249

Kieferle, J. (2015). "Bim Interactive - about Combining Bim and Virtual Reality - a Bidirectional Interaction Method for Bim Models in Different Environments," in Proceedings of the 33rd eCAADe Conference (Vienna, Austria), 69

Kilteni, K., Groten, R., and Slater, M. (2012). The Sense of Embodiment in Virtual Reality. Presence. 21, 373-387. doi:10.1162/pres_a_00124

Lombard, M., and Ditton, T. (1997). At the Heart of it All: The Concept of Presence. J. Computer-Mediated Commun. 3.

Robert McNill and Associates (2019). Compute Guides.

Michalos, G., Karvouniari, A., Dimitropoulos, N., Togias, T., and Makris, S. (2018). Workplace Analysis and Design Using Virtual Reality Techniques. CIRP Ann. 67, 141-144. doi:10.1016/j.cirp.2018.04.120

[Dataset] Mindesk (2019a). Mindesk - Multiuser Vr in Rhinoceros.

[Dataset] Mindesk (2019b). Mindesk Rhino Link.

Moubile, M. (2018). Towards Real-Time Parametric Structural Modeling in Virtual Reality Using a Game Engine. Master's Thesis. Ghent, Belgium: Ghent University,Faculty of Engineering and Architecture.
Oh, C. S., Bailenson, J. N., and Welch, G. F. (2018). A Systematic Review of Social Presence: Definition, Antecedents, and Implications. Front. Robot. AI. 5, 114 doi:10.3389/frobt.2018.00114

Quinn, G., Galeazzi, A., Schneider, F., and Gengnagel, C. (2018). "Structvr Virtual Reality Structures," in Proceedings of the Symposium of the International Association for Shell and Spatial Structures (Boston), 1-8.

Reisinger, J., Hollinsky, P., and Kovacic, I. (2021a). Design Guideline for Flexible Industrial Buildings Integrating Industry 4.0 Parameters. Sustainability. 13, 10627. doi:10.3390/su131910627

Reisinger, J., Zahlbruckner, M., Kovacic, I., Kán, P., and Wang-Sukalia, X. (2021b). "Framework Proposal for Automated Generation of Production Layout Scenarios: A Parametric Design Technique to Connect Production Planning and Structural Industrial Building Design," in 2021 European Conference on Computing in Construction.11

Reisinger, J., Knoll, M., and Kovacic, I. (2020). "Parametric Structural Design for Automated Multi-Objective Optimization of Flexible Industrial Buildings," in Proceedings of the 37th International Symposium on Automation and Robotics in Construction (ISARC). Editor F. H. T. K. Osumi Hisashi (International Association for Automation and Robotics in Construction IAARC). doi:10. $22260 /$ isarc $2020 / 0028$

Ruddle, R. A., and Lessels, S. (2009). The Benefits of Using a Walking Interface to Navigate Virtual Environments. ACM Trans. Comput.-Hum. Interact. 16 (1). doi:10.1145/1502800.1502805

Sampaio, A. Z. (2018). "Enhancing Bim Methodology with Vr Technology," in State of the Art Virtual Reality and Augmented Reality Knowhow (Rijeka: IntechOpen). chap. 5. doi:10.5772/intechopen.74070

Sidani, A., Matoseiro Dinis, F., Duarte, J., Sanhudo, L., Calvetti, D., Santos Baptista, J., et al. (2021). Recent Tools and Techniques of Bim-Based Augmented Reality: A Systematic Review. J. Building Eng. 42, 102500. doi:10.1016/j.jobe.2021.102500

Slater, M., Usoh, M., and Steed, A. (1994). Depth of Presence in Virtual Environments. Presence: Teleoper. Virtual Environ. 3, 130-144. doi:10.1162/ pres.1994.3.2.130

Warwick, K., Gray, J., and Roberts, D. (1993). Virtual Reality in Engineering. Institution of Electrical Engineers.

Whyte, J. (2003). Industrial Applications of Virtual Reality in Architecture and Construction. Spec. Issue Virtual Reality Technology Architecture Construction. $8,43-50$

Wolfartsberger, J., Zenisek, J., and Sievi, C. (2018). Chances and Limitations of a Virtual Reality-Supported Tool for Decision Making in Industrial Engineering. IFAC-PapersOnLine. 51, 637-642. doi:10.1016/j.ifacol.2018.08.390

Zaker, R., and Coloma, E. (2018). Virtual Reality-Integrated Workflow in BimEnabled Projects Collaboration and Design Review: a Case Study. Visualization Eng. 6, 15. doi:10.1186/s40327-018-0065-6

Zhao, T., and Tseng, C.-L. (2003). Valuing Flexibility in Infrastructure Expansion. J. Infrastructure Syst. 9, 89-97. doi:10.1061/(asce)1076-0342(2003)9:3(89)

Conflict of Interest: The authors declare that the research was conducted in the absence of any commercial or financial relationships that could be construed as a potential conflict of interest.

Publisher's Note: All claims expressed in this article are solely those of the authors and do not necessarily represent those of their affiliated organizations, or those of the publisher, the editors and the reviewers. Any product that may be evaluated in this article, or claim that may be made by its manufacturer, is not guaranteed or endorsed by the publisher.

Copyright (๑ 2022 Podkosova, Reisinger, Kaufmann and Kovacic. This is an openaccess article distributed under the terms of the Creative Commons Attribution License (CC BY). The use, distribution or reproduction in other forums is permitted, provided the original author(s) and the copyright owner(s) are credited and that the original publication in this journal is cited, in accordance with accepted academic practice. No use, distribution or reproduction is permitted which does not comply with these terms. 